\title{
IDENTIFICACIÓN DE LOS SISTEMAS DE SUMINISTRO Y DISPOSICIÓN DEL AGUA EN UNA COMUNIDAD RURAL
}

\author{
IDENTIFICATION OF WATER SUPPLY AND DISPOSAL \\ SYSTEMS IN A RURAL COMMUNITY
}

\author{
Laura V. Morales-Tejeiro•laura.morales@unillanos.edu.co \\ Maestría en Gestión Ambiental Sostenible, Universidad de los Llanos, Villavicencio-Colombia.
}

Juan M. Trujillo-González • trujillo@unillanos.edu.co

PhD. Química agrícola, Universidad de los Llanos, Villavicencio-Colombia.

Marco A. Torres-Mora•marcotorres@unillanos.edu.co

PhD. Tecnologías energéticas y ambientales para el desarrollo, Universidad de los Llanos, Villavicencio-Colombia.

Citación: Morales-Tejeiro, L. V., Trujillo-González, J. M., Torres-Mora, M. A. (2022). Identificación de los sistemas de suministro y disposición del agua en una comunidad rural. Revista de Investigación Agraria y Ambiental, 13(1), 205 - 220. DOI: https://doi.org/10.22490/21456453.4271

\section{RESUMEN}

Contextualización: El agua es el recurso natural de mayor importancia. No obstante, su aprovechamiento es limitado, ya que sólo el $1 \%$ del agua del planeta es potencialmente apto para el consumo humano. En áreas rurales los sistemas de abastecimiento y conducción de aguas residuales pueden ser considerados artesanales o rudimentarios, lo cual no garantiza el flujo constante del recurso ni la calidad óptima para el consumo humano. Además, tiene efectos negativos para la salud pública y genera afectación a los recursos naturales.

Vacío de conocimiento: En este sentido, este tipo de trabajos cobran importancia al describir los mecanismos asociados al recurso hídrico con los que cuentan las comunidades rurales, para que esta información, a su vez, contribuya en la formulación de propuestas desde los grupos de interés.

Propósito: El presente estudio tuvo como objetivo principal identificar los sistemas de abastecimiento de agua y sistemas de disposición de aguas residuales de la comunidad rural de la vereda Barcelona, en el municipio de Villavicencio (Meta).

Metodología: La información analizada obtuvo por medio de encuestas semiestructuradas que fueron realizadas de manera aleatoria en distintos sectores de la vereda.

Resultados y conclusiones: Entre los principales resultados se encontró que la comunidad se abastece de agua por medio de aljibes (100\%) y realiza la disposición de aguas residuales mediante cuatro sistemas: pozo séptico propio (44 $\%)$; pozo séptico comunitario (20\%); pozo séptico propio y campo abierto (28 \%); pozo séptico comunitario y campo abierto (8\%). Adicionalmente, la percepción de la calidad del agua varío de acuerdo con el género. El $100 \%$ de las mujeres identificaron como de baja calidad el agua para consumo humano, y en razón de esto se abastecen de fuentes seguras para su consumo y preparación de alimentos; mientras que los hombres la identificaron como regular y el $37 \%$ la consumen directamente. Finalmente, se evidenció que el actual mecanismo de limpieza de los sistemas de disposición de aguas residuales puede convertirse en un factor de riesgo de contaminación y afectación a la salud pública.

Palabras Clave: recurso hídrico; sistema de abastecimiento; aguas residuales; comunidad rural 


\section{ABSTRACT}

Contextualization: Water is the most important natural resource, however, its use is limited, since only $1 \%$ of the water on the planet is potentially suitable for human consumption. In rural areas, the systems for supplying and wastewater disposal can be artisan or rudimentary, which does not guarantee the constant flow of the resource, the optimal quality for human consumption, and has negative effects on public health and impact on natural resources.

Knowledge gap: In this sense, this type of studies becomes important to describe the mechanisms associated with water resources that rural communities have, so this information, in turn, contributes to the formulation of proposals from interest groups.

Purpose: The main objective of this study was to identify the water supply systems and wastewater disposal systems in the rural community of the Vereda Barcelona, in the municipality of Villavicencio (Meta).

Methodology: The analyzed information was obtained through semi-structured surveys that were carried out randomly in different sectors of the village.

Results and conclusions: Among the main results, it was found that the community is supplied with water through cisterns (100 $\%$ ) and disposes of wastewater through four systems: its own septic well (44\%), community septic well (20\%), septic well own and open field (28\%), and community septic well and open field ( $8 \%)$. Also, the perception of water quality varied according to gender. $100 \%$ of the women identified the water as low quality for human consumption, and because of this, they are supplied from safe sources for their consumption and food preparation; while men identified it as regular and $37 \%$ consume it directly. Finally, it was evidenced that the current cleaning mechanism for wastewater disposal systems can become a risk factor for contamination and affection on public health.

Keywords: water resource, water supply system, wastewater, rural community

\section{RESUMEN GRAFICO}
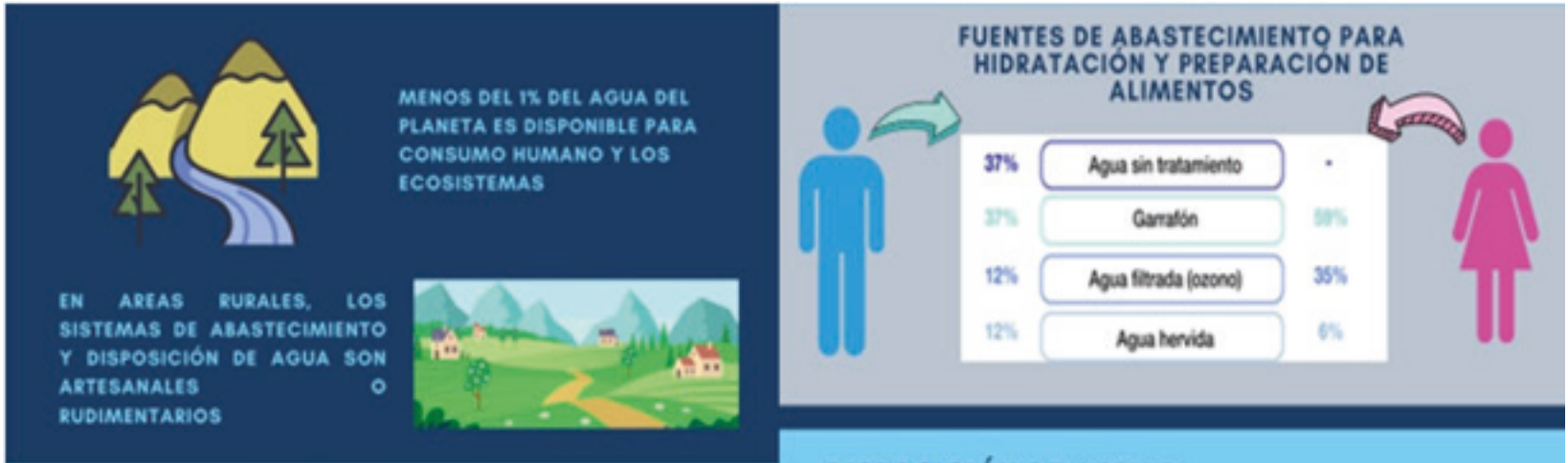

VEREDA BARCELONA

SISTEMAS DE ABASTECIMIENTO

DISPOSICIÓN DE AGUAS RESIDUALES

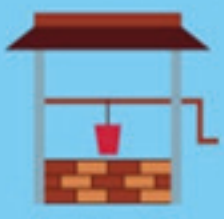

LA COMUNIDAD SE ABASTECE DE AQUA POR MEDIO DE ALIBES (100\%).

- pozo st́ptico propo (44\%).

- pozo séptico comunitavio (20\%)

- pozo strtico nopio $r$ CAMPO ABItRTO (208)

- pozo síptico comunitaeio Y CAMPO ABIERTO (OS)

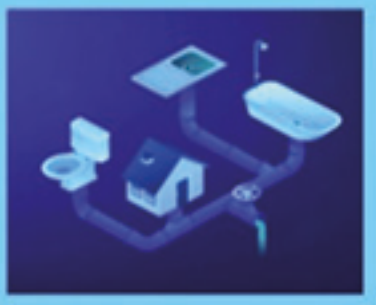

Fuente: autores 


\section{INTRODUCCIÓN}

El agua es el recurso natural de mayor importancia para la supervivencia humana. Soporta diferentes procesos que permiten el desarrollo apropiado de las sociedades, así como también tiene una alta importancia para el sostenimiento de las funciones ecológicas de los sistemas naturales ( Fernández, 2012). Es un recurso limitado porque, de toda el agua del planeta, solo $1 \%$ es potencialmente apto para el consumo humano y alguna parte de esta porción está en regiones con conflictos 0 contaminadas Iñiguez, et al., 2015). Por otra parte, los recursos hídricos aún siguen siendo de difícil acceso para algunas comunidades en Latinoamérica, pues (hacia el 2015) solo un $65 \%$ de la población tenía acceso a servicios de agua potable gestionados de forma segura, aproximadamente 25 millones de personas no contaban con acceso a un servicio básico de agua y 222 millones no tenían servicios de agua potable gestionados de forma segura ( Organización de las Naciones Unidas para la Educación, la Ciencia y la Cultura [UNESCO], 2019).

En este sentido, la crisis por la disponibilidad de agua, para el soporte de las actividades domésticas, aumenta cada vez más en algunas regiones del mundo, principalmente por la creciente demanda para uso agrícola e industrial y por los efectos del cambio climático (Delgado-García et al., 2017). En América Latina el escenario es crítico. Los gobiernos tienen limitantes económicas, institucionales y climáticas que no les permite garantizar completamente las necesidades básicas de la población (PinillaRodríguez y Torres-Sánchez, 2019). Además, la crisis se agudiza por el alto deterioro ambiental de los ecosistemas; generado como consecuencia de una sobreexplotación del recurso hídrico, por las extracciones de agua para el desarrollo de las actividades productivas o porque las aguas son utilizadas como vehículo para eliminar las aguas residuales de la población (Trujillo-González et al., 2017). Por su parte, los factores sociales, culturales y políticos limitan la inversión o la capacidad técnica necesaria para gestionar el recurso hídrico ( Padrón y Cantú, 2015).

La problemática de acceso a fuentes de agua, con la calidad y disponibilidad óptima para abastecimiento humano, es un tema de vital importancia y debe estar en las agendas de las autoridades como de la sociedad en general (Faviel et al., 2018). Colombia es un territorio con abundancia hídrica, considerado el sexto país en el mundo con mayor disponibilidad de agua, y con alrededor del $5 \%$ del agua dulce superficial del planeta (Organización Mundial de las Naciones Unidas para la alimentación y la agricultura [FAO], 2020). Sin embargo, la disponibilidad del agua no es homogénea en el territorio nacional (Díaz, 2019); el estado de la oferta de agua ha sido resultado de los modelos asumidos por el Estado colombiano, vinculados con la minería, la agricultura extensiva y los desarticulados planes de expansión urbana (Mejía, 2004; Bermúdez, 2014).

En el plano rural, las propias comunidades son los actores principales y quienes jalonan la gestión del recurso. Se adaptan a las particularidades propias del entorno, donde se apropian de modelos tecnológicos y se organizan con el objeto de acceder al recurso. Este trabajo en conjunto es resultado de las relaciones sociales y del tejido social que se logra construir en función del agua, orientado por líderes con capacidad para comunicar y transmitir sus experiencias (Delgado-García et al., 2017; Quintana, 2014). No obstante, las estrategias no garantizan abastecimiento a largo plazo ni la calidad en los términos establecidos por las normas para el caso colombiano, reglado por la resolución 2115 de 2007 (Ministerio de la protección social, 2007).

Con base en lo anterior, la Vereda Barcelona de la ciudad de Villavicencio se muestra como un escenario idóneo para la evaluación de la gestión del recurso hídrico, es un sector rural donde el recurso es gestionado por la comunidad y además está en una etapa de crecimiento demográfico y de urbanización (Barrera, 2012; Alcaldía de Villavicencio, 2015 b). En este sentido, el presente estudio tiene como objetivo principal "identificar los sistemas de abastecimiento y disposición del agua en la comunidad rural de la Vereda Barcelona", a partir de encuestas hechas en la comunidad misma, y que esta información se convierta en un fundamento para la formulación de una herramienta de gestión ambiental eficaz, y para la formulación de planes y proyectos encaminados a la gestión integral del recurso hídrico, desde las comunidades o las instituciones. 


\section{MATERIALES Y MÉTODOS}

\section{1. Área de Estudio}

La vereda Barcelona se ubica en el municipio de Villavicencio (Meta, Colombia), a $86 \mathrm{~km}$ de la capital del país. Hace parte de las 61 veredas rurales del municipio, se ubica en el corregimiento siete, el cual cubre un área total de 40345,27 Ha, ocupando así el $31,01 \%$ del territorio municipal. La vereda cuenta con un área de 2465 hectáreas y se encuentra ubicada a una altura promedio de 400 m. s. n. m (Alcaldía de Villavicencio, 2015a). El clima es típicamente cálido, con temperaturas promedio de $25^{\circ} \mathrm{C}$, posee una temperatura inferior al casco urbano del municipio. En este sector es común encontrar bosques húmedos tropicales en transición y bosques de galería con grandes porciones de tierra. Está irrigado por numerosas fuentes hídricas, algunas altamente intervenidas ( Trujillo-González et al., 2015). La ubicación del área de estudio se encuentra especificada en la Figura 1; Las fotografías áreas se obtuvieron por medio de Google Earth y los mapas se realizaron por medio del programa ArcGis.

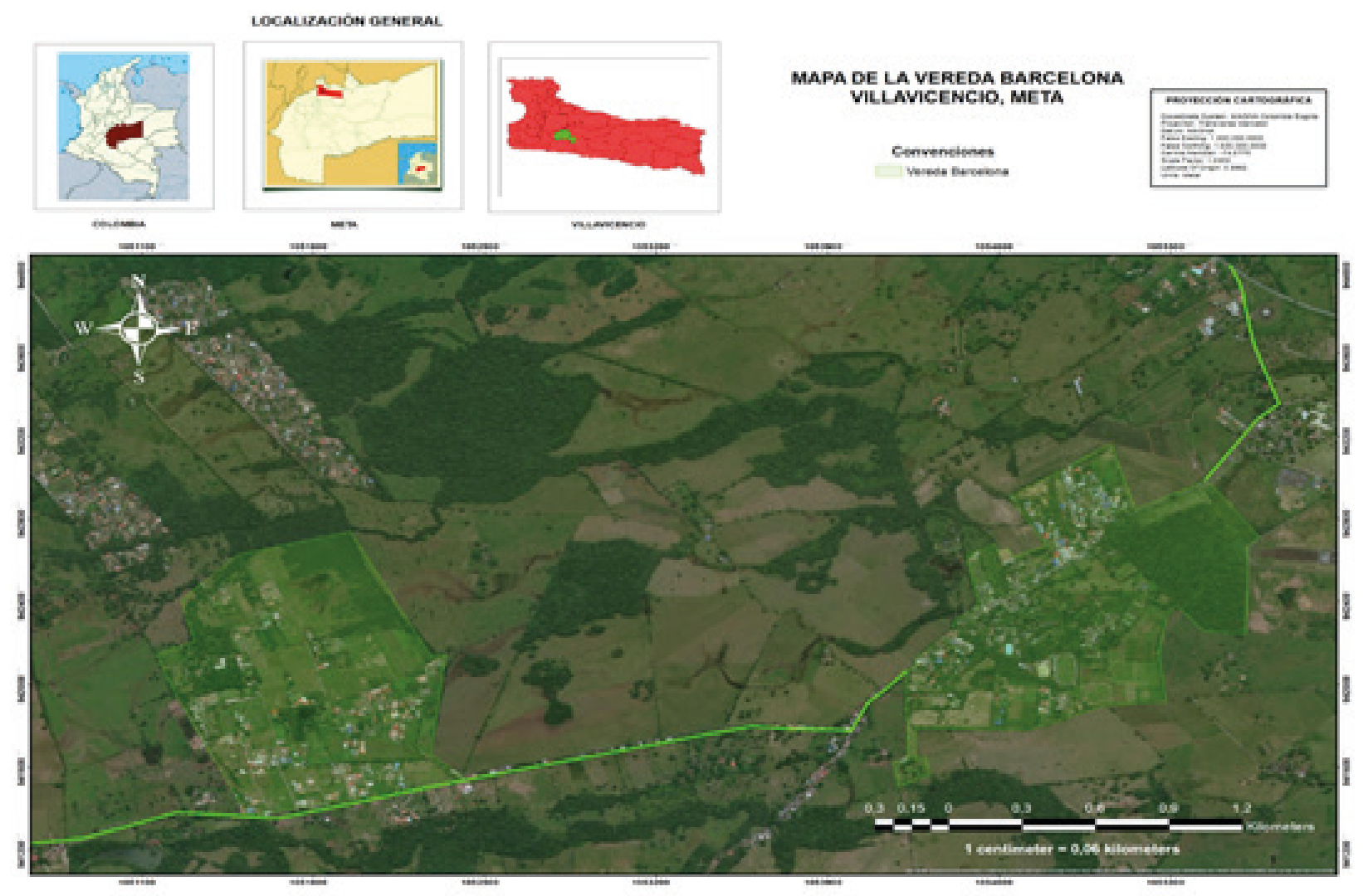

Figura 1. Ubicación geográfica de la vereda Barcelona, Villavicencio-Colombia.

Fuente: Autores

\subsection{Recolección de información}

En la recolección de la información primaria se empleó el muestreo probabilístico de tipo aleatorio simple (Otzen y Manterola, 2017), la unidad de análisis fueron las viviendas, y el tamaño de la muestra se determinó de acuerdo con el número total de viviendas ubicadas en la Vereda (150 viviendas), cada una con 4-5 personas aproximadamente. Las condiciones de inclusión en el estudio fueron: vivir por más de cinco (5) años en la vereda
Barcelona y ser mayor de edad. Establecidos estos requisitos se lograron encuestar 25 hogares que cumplían con esta condición. La información secundaria, por su parte, se tomó de publicaciones, informes académicos e institucionales.

El instrumento metodológico que se utilizó fue la encuesta (Anexo 1), la cual se basó en escalas de intensidad o percepción, con preguntas en abanico y dicotómicas (López y Fachelli, 2015). Estas escalas permitieron 
identificar la percepción social sobre la calidad del agua de la vereda Barcelona. Previamente se realizó una prueba piloto donde se aplicaron encuestas en campo, posteriormente se ajustó el cuestionario y se aplicó la encuesta final en las viviendas.

\subsection{Análisis de Datos}

La intención de las encuestas fue de carácter exploratorio para identificar los fenómenos relevantes que suceden en la vereda Barcelona frente a la gestión del agua. Con este propósito, la información obtenida se organizó en tablas con el fin de relacionar las variables cualitativas (género, nivel educativo, tenencia de la tierra, tipo de sistema de suministro y disposición de agua, percepción de la calidad del agua, entre otros) con las variables cuantitativas (profundidad del aljibe, distancia aljibe pozo séptico, cantidad de cloro para tratamiento y frecuencia, entre otros). Posteriormente, la población encuestada fue separada de acuerdo diferentes categorías analíticas: género, nivel educativo y tipo de tenencia de la tierra.

Para comparar cada una de las categorías analíticas, se usaron como base los cálculos de los porcentajes de las frecuencias obtenidas en cada una de las variables cualitativas de interés (sistemas de suministro y disposición, percepción de la calidad del agua) y se realizó un análisis tabular, en el cual se relacionaron las variables de clasificación de la población y las variables de gestión del agua a partir de las respuestas de la encuesta. Con base en estas respuestas se construyeron categorías analíticas de acuerdo con la metodología empleada por Villamagua (2017). Adicionalmente, para el análisis cuantitativo se tuvieron en cuenta la frecuencia de las respuestas relacionadas a una o más de las categorías analíticas determinadas. Posteriormente, la población encuestada fue separada de acuerdo diferentes categorías analíticas: género, nivel educativo y tipo de tenencia de tierra.

Para comparar cada una de las categorías analíticas, se usaron como base los cálculos de los porcentajes de las frecuencias obtenidas en cada una de las variables cualitativas de interés (género, nivel educativo, tenencia de la tierra, tipo de sistema de suministro y disposición de agua, percepción de la calidad del agua, entre otros) con las variables cuantitativas (profundidad del aljibe, distancia entre pozo séptico y aljibe, cantidad de cloro para tratamiento y frecuencia, entre otros), como se muestra en la figura 2; en la que se realiza la comparación entre la variable cualitativa (nivel de educación) frente a la variable cuantitativa (distancia entre pozo séptico y aljibe).

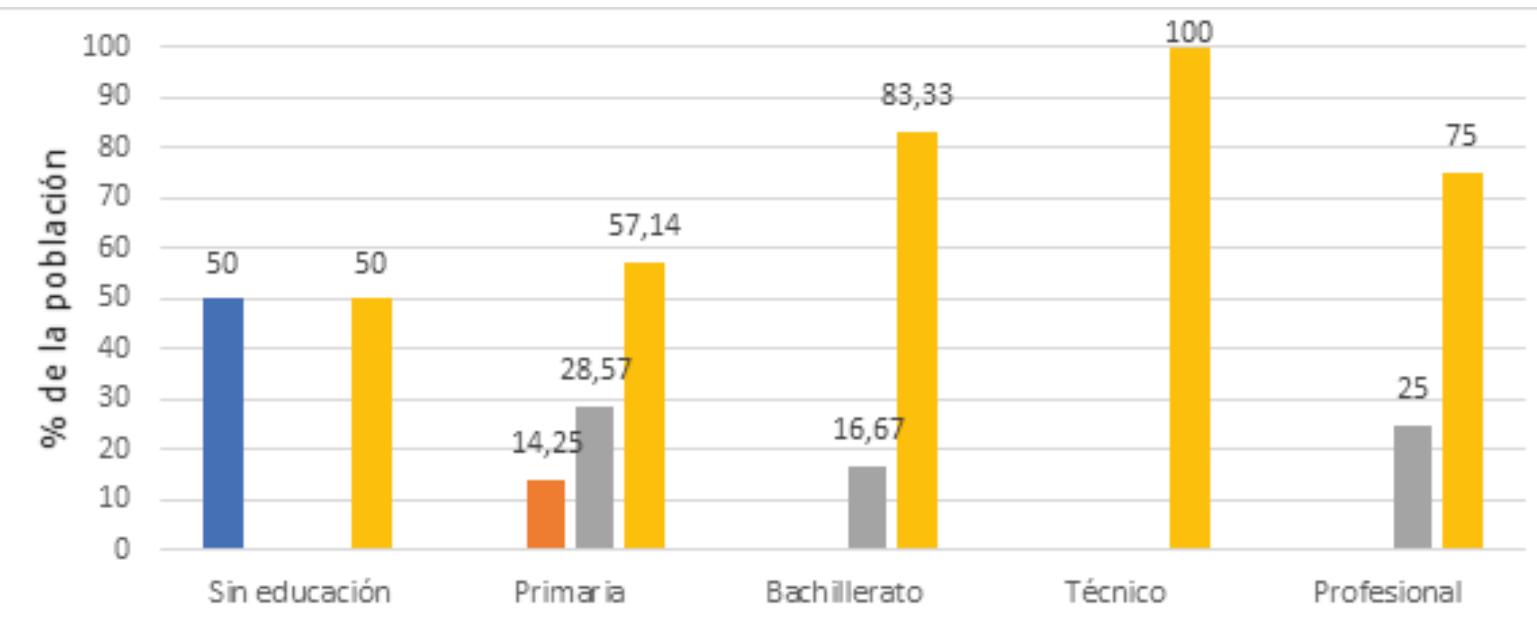

Distancia entre pozo séptico y aljíbe

- 1 a $3 \mathrm{~m} \quad 4 \mathrm{a} 6 \mathrm{~m} \quad 7 \mathrm{a} 9 \mathrm{~m}$ mas de $9 \mathrm{~m}$

Figura 2. Nivel de Educación vs distancia entre el pozo séptico y aljibe.

Fuente: Autores 


\section{RESULTADOS Y DISCUSIÓN}

\subsection{Generalidades}

Del total de la población encuestada, el $47 \%$ de las mujeres tenía una edad en el rango de 41 a 60 años, mientras que el $37 \%$ de los hombres fueron mayores de 60 años. La ocupación de los encuestados fue principalmente "comerciante" (52 \%) y el negocio predominante, las tiendas de víveres; seguido por personas que se dedican a las labores del hogar (32\%); finalmente, un $12 \%$ de los encuestados se clasificó como estudiante que cursa programas profesionales en la sede Barcelona de la Universidad de los Llanos. Otras actividades que han sido desarrolladas en el sector, en el pasado, corresponden a actividades agrícolas y pecuarias, según (Trujillo-González et al., 2015) . No obstante, en el presente trabajo ninguno de los encuestados manifestó dedicarse a dicha actividad.

En cuanto a la antigüedad en la zona, se encontró que el $24 \%$ de la población reside en la vereda hace más de 20 años y el $68 \%$ cuenta con vivienda propia. En lo referente al uso del suelo, el $72 \%$ de las viviendas son de uso residencial únicamente, frente al $28 \%$ que son utilizadas para uso residencial y comercial con establecimientos que se dedican a la venta de alimentos y licores, los cuales se benefician directamente de los habitantes del sector, quienes son sus principales clientes. Esto último coincide con lo mencionado por Caro-Caro et al. (2014), quien señala que en el sector se encuentra infraestructura recreativa como piscinas, parques de juegos y otros, cuyo uso es de tipo turístico. Por otro lado, la información disponible de la vereda Barcelona indica otros usos del suelo relacionados con actividades productivas, como cobertura de pasto para ganadería, presencia de piscícolas y siembra de diversos cultivos (Caro-Caro et al., 2014).

Así mismo, se evidencian procesos de expansión y un cambio en el uso del suelo, de agrícola a comercial y turístico, notándose la presencia de nueva infraestructura, como edificios habitacionales, que cambian la configuración del paisaje rural hacia uno de tipo urbano (Barrera, 2012). Esta expansión se evidencia en la información presentada por el Plan de Ordenamiento Territorial 2015, el cual encontró diferentes factores que han incidido en el proceso de crecimiento urbano. Además, en este mismo plan se menciona que parte de la vereda se enfrenta a una reorganización de su infraestructura, a causa de la construcción de la nueva vía Villavicencio - Acacias. Esta construcción es de tal magnitud que generará cambios en el paisaje (Alcaldía de Villavicencio, 2015a).

Ochoa (2017) menciona que el proceso de urbanización de la vereda Barcelona es consecuente con las tendencias de desarrollo territorial en la ciudad de Villavicencio, que están llevando a una creciente penetración del suelo urbano al suelo rural y cuyo proceso coincide en la construcción de viviendas y fincas turísticas que no cumplen con la normatividad estipulada para suelo rural. Estos predios están ocasionando transformaciones en la vocación productiva, y posible deterioro ambiental por la reducción de bosques y fuentes hídricas. Lo anterior se respalda con la información obtenida en las encuestas, en las cuales la comunidad manifestó que, desde que está la sede Barcelona de la Universidad de los Llanos en el sector, el crecimiento poblacional de la vereda ha sido considerable y ha estado acompañado de la construcción de edificios para la vivienda de estudiantes y otras actividades comerciales, relacionadas con servicios ofrecidos a esta misma población, tales como: piscinas, billares, canchas de tejo, papelerías, restaurantes, canchas de futbol, entre otros.

\subsection{Sistemas de abastecimiento de agua}

Al igual que en el resto del mundo, en Colombia el agua y su disponibilidad se encuentran amenazadas por su inadecuado uso, lo cual implica retos determinantes ante el saneamiento y la higiene de la población y los ecosistemas vinculados (Programa de Las Naciones Unidas Para El Desarrollo- [PNUD], 2018). Además, en el país es notorio la escasa información al respecto, ya que parte de esta no se encuentra registrada en varios municipios.

Según la Contraloría General de la Nación (2018), el número de municipios que reportaron cobertura en acueducto en un $100 \%$ disminuyó de 162 a 90, en el periodo comprendido del 2010 al 2017. En particular 
en el municipio del Meta, acorde con lo evidenciado por el Programa de las Naciones Unidas para el Desarrollo en el informe "Meta, Retos y Desafíos para el Desarrollo Sostenible" (PNUD, 2020), el departamento tiene una cobertura del $93 \%$ de disponibilidad de agua potable. Un $59 \%$ de ella es proveída directamente del acueducto, pero el índice de riesgo de calidad del agua es de $32 \%$ lo que significa un riesgo medio para su consumo. En este sentido, la ciudad de Villavicencio tiene un promedio de $86 \%$ de cobertura en la cabecera municipal (Castro-Garzón et al., 2013). La realidad de la vereda Barcelona es otra, en el total de los hogares entrevistados el abastecimiento es soportado por pozos profundos o aljibes y en los casos en los que se considera que el agua del aljibe no es apta para el consumo humano, se proveen de agua embotellada para la hidratación y preparación de alimentos.

Según Vélez et al., (2011), los aljibes se utilizan para abastecer de agua a pequeñas comunidades, mediante el uso de bombas manuales o sistemas de bombeo muy simples para extraer el recurso hídrico. Este tipo de sistema de abastecimiento consta de pozos que se excavan de manera manual, con profundidades entre 5 y 10 metros y diámetros de hasta un metro en promedio. Adicionalmente, a la estructura interna de los pozos suele hacérsele un recubrimiento de tubo PVC u otro material, con el objeto de evitar su derrumbamiento y la posibilidad de contaminar las aguas subterráneas. De acuerdo con este parámetro estructural, dentro de los hogares encuestados el 60 $\%$ de los pozos presentaban algún tipo de recubrimiento (Cemento, $52 \%$; PVC, 4 $\%$; y Baldosa, $4 \%$ ), mientras que el $40 \%$ restante no contaban con ningún tipo de reforzamiento.

En el estudio se estableció, también, una relación entre el uso de la tierra y el material de construcción del aljibe; como se mencionó antes, se encontraron viviendas destinadas para uso residencial y viviendas de uso residencial - comercial. De acuerdo con esto se encontró que el $66 \%$ de los aljibes de las viviendas de tipo residencial se encuentran recubiertos por tubos de cemento, el $27 \%$ son rústicos y el $5 \%$ en tubo PVC. En el caso de las viviendas de uso residencialcomercial, el $71,4 \%$ de los aljibes se encuentran construidos de manera rustica; $14,29 \%$, recubiertos por tubo de cemento; y el $14,29 \%$ restante, recubiertos en baldosa (figura 3).

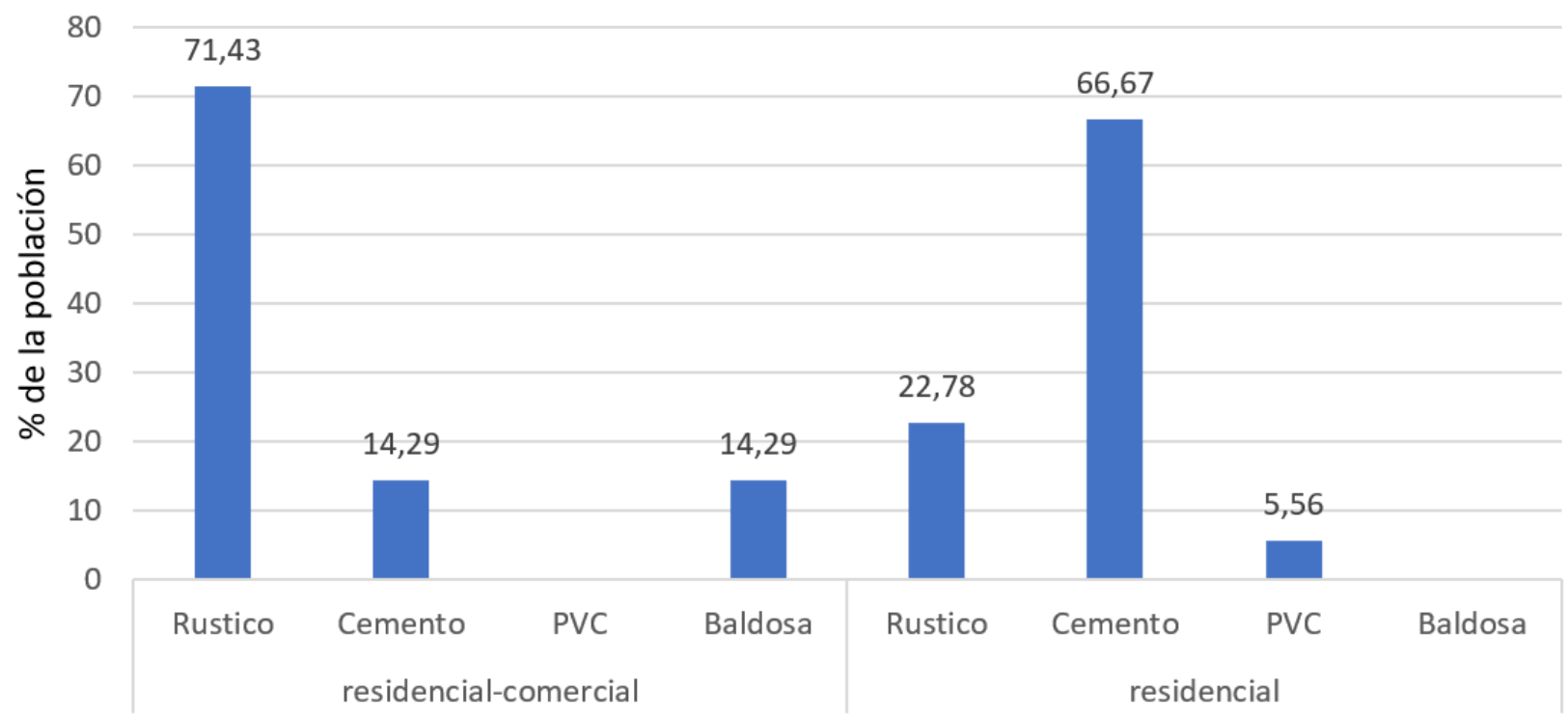

uso de la tierra

Figura 3. Material de recubrimiento del aljibe en relación con el uso de la tierra. 
El agua que se extrae de estos pozos llega a un tanque elevado, una vez almacenada se distribuye para las diferentes labores del hogar o en algunas ocasiones para consumo humano. Como medidas de tratamiento y aseguramiento de la calidad del agua, el $84 \%$ de los pozos son tratados mediante el suministro de dos pastillas de cloro de $200 \mathrm{~g}$ al año; esta cantidad es superior a la recomendada por la Organización Mundial de la salud, (2004), la cual dice que la concentración del cloro residual en el agua debe estar entre 0,2 y 0,5 mg/l por día, por lo que la cantidad necesaria para tratar las aguas de aljibes con las dimensiones de los encontrados en la vereda, que poseen un volumen de 15,700 litros aproximadamente, sería de 3,14 a 7,85 gramos de cloro libre o residual.

De acuerdo con esta particularidad, es importante que la comunidad conozca las propiedades de las aguas del aljibe para formular la dosificación de cloro. En este sentido, se recomienda realizar ensayos a nivel de laboratorio para determinar la dosis óptima, la periodicidad del desinfectante y las condiciones adecuadas de operación para la desinfección (Ministerio de Desarrollo Económico, 2000). Por otro lado, el Instituto Nacional de Tecnología Agropecuaria (2014) establece que la periodicidad con la que se debe aplicar tratamiento con cloro, en el caso de aljibes, debe ser de 30 a 45 días, pues las condiciones de oscuridad y temperatura que se genera en el interior de un aljibe contribuyen a preservar la capacidad de desinfección de este elemento. Con respecto a esto, se encontró que en la vereda hubo diferencias: el $80 \%$ de los encuestados aplican pastillas desinfectantes en un periodo de cada 6 meses a un año, un $4 \%$, cada dos años o más y un $16 \%$ carecen de insumos para la cloración. Estos últimos mencionaron que prefieren mantener el pozo cerrado en la parte superior para evitar que caigan partículas que puedan contaminarlo.

Esta investigación evidenció que la población valora la estacionalidad por el efecto que tienen las aguas de lluvia sobre la calidad y cantidad del agua. Sobre esto, mencionan que en épocas de mayor pluviosidad identifican el aumento del nivel de agua en el aljibe, así como la disminución de la turbidez del agua, ya que se observa un agua más "clara". El periodo crítico por escasez de lluvia en la cuenca del río Ocoa suele presentarse en los meses de enero, febrero y marzo ( Instituto de Hidrología meteorología y Estudios Ambientales de Colombia [IDEAM], 2020), es decir, la precipitación es baja y se disminuyen los caudales en los cauces de los ríos y quebradas.

Lo anterior se respalda con los resultados de precipitación de los últimos 33 años de la estación meteorológica de la Universidad de los Llanos, sede Barcelona, que señala el periodo de diciembre a febrero como el que tiene el promedio de precipitaciones mensuales más bajo, con valores de precipitación inferiores a $120 \mathrm{~mm}$, por lo que es de esperarse de que en esta época el recurso sea más escaso y de menor calidad. Contrario a esto, los meses de abril, junio y julio son los de mayor precipitación (IDEAM, 2020), por lo que allí no solo se nota una mejora en la obtención del recurso sino que se muestra cómo un escenario clave para el desarrollo de estrategias para la obtención y almacenamiento del agua en épocas de escasez. En cuanto a esto, según los datos de la estación meteorológica, los meses con mayor pluviosidad han sido abril, mayo y junio con valores superiores a $460 \mathrm{~mm}$.

A pesar de que la población identifica esta situación de variabilidad climática en la disponibilidad del recurso, no se evidenció estrategia alguna en cuanto a los cambios en el uso rutinario del agua de acuerdo con la época. Del mismo modo, no mostraron tener un plan de almacenamiento concreto, pues manifiestan que, a pesar de que el nivel del agua disminuye en los pozos en época seca, nunca han sufrido de escasez. Esta situación presenta una oportunidad para que la comunidad se organice en cuanto al uso que se le está dando al recurso, es decir, para identificar las actividades comerciales y/o domesticas a las que se está destinando el mismo y a cuantificar cuanto de este se consume en cada una de estas actividades desarrolladas en la vereda.

Lo ideal es que las condiciones del acceso al agua sean equitativas para todos los actores que se beneficien del recurso, así que los intereses económicos no deberían 
prevalecer sobre los intereses comunes en el momento de decidir las actividades prioritarias. Ante esta situación, Martín y Justo (2015), proponen que deben propiciarse mecanismos institucionales que fomenten la cohesión y pertenencia social, diferentes de la sanción o la responsabilidad y más centrados en la prevención, participación y racionalidad de la acción cooperativa. Con base en todo lo anterior, la gobernanza del agua como método de abastecimiento, uso y manejo del recurso hídrico es un método imparcial en cuanto a edad, género - capacidad económica. De acuerdo con Gómez y Collazos (2015), el crecimiento poblacional, cercano a las ciudades, afecta las condiciones ambientales. Entre las consecuencias ambientales ocasionadas por esta situación se encuentran: contaminación del aire, contaminación auditiva, invasión de las tierras fértiles o que no son aptas para la construcción de viviendas y aumento de la contaminación y presión sobre el recurso hídrico. En la población de la Vereda Barcelona, específicamente, se identificó que el problema ambiental de la contaminación del agua, por fuentes externas, obedece a la falta de mantenimiento del pozo séptico comunitario. Por otra parte, la falta de mantenimiento de los pozos profundos 0 aljibe es otro factor que afecta la calidad del recurso.

Según Chávez et al. (2013), una de las desventajas de los pozos profundos - aljibes es que el nivel freático puede estar contaminado por filtración de aguas residuales. Las sustancias contaminantes se infiltran (como consecuencia de las actividades que se realizan en la superficie del terreno) y se desplazan hasta el nivel del agua de los pozos profundos o aljibes; cuando llegan al agua, estas sustancias se mueven con ella y se pueden trasladar de un pozo profundo a otro Pulido et al., 1997). Desde este punto de vista, se puede determinar que la mayoría de los problemas que enfrenta el recurso hídrico subterráneo son causados por el hombre. Por otra parte, la ausencia de sistemas de acueducto en zonas rurales obliga a la comunidad a buscar diferentes formas de transporte, abastecimiento y almacenamiento del agua, afectando así su calidad microbiológica y convirtiéndola en un riesgo para la salud del consumidor
( Reales et al., 2014). Esto coincide con la percepción que tiene los habitantes de la Vereda Barcelona, los cuales consideran que la calidad del agua de los pozos profundos o aljibes es de calidad regular.

Ante esto, según lo evidenciado por Venegas et al. (2014), la frecuente limpieza de los lugares de almacenamiento de agua, mantener cubiertos los aljibes, realizar su limpieza y desinfección periódicamente, además de optar buenas prácticas de higiene dentro de los hogares, reduce de forma significativa la aparición de microorganismos. Del mismo modo, limpiar los tanques elevados o lugares de almacenamiento de agua y lavarse las manos de manera adecuada antes de realizar cualquier tipo de actividad que involucre tener contacto con el agua almacenada, mantiene la calidad microbiológica del recurso (Brick et al., 2004).

\subsection{Percepciones sobre la calidad del agua}

La percepción de la calidad del agua por parte de los usuarios es un tema que puede estar relacionado con la salud pública, pues es a partir de este criterio que los usuarios determinan los usos que se dan al líquido obtenido. De acuerdo con la opinión de los entrevistados, la percepción de la calidad tiene diferencias evidentes según el género. La mayoría de las mujeres percibieron que la calidad del agua proveniente del pozo profundo o aljibe es mala, por lo que un $59 \%$ de ellas prefiere comprar agua embotellada en "garrafones"; el 35 \% escoge utilizar un sistema de tratamiento de agua (filtros de ozono); y el $6 \%$ restante, hervir el agua para consumo humano. Contrario a esto, los hombres entrevistados manifestaron que el agua tiene una calidad regular $(67 \%)$ y buena (33\%), de manera que el $37 \%$ de los hombres consideró que esta agua se puede utilizar para consumo humano sin ningún tipo de tratamiento, por lo que es probable que esta población este expuesta a riesgos de salud (figura 4).

La presente investigación obtuvo, en cuanto al género, que las mujeres mostraron tener un mayor conocimiento ambiental que los hombres, lo cual pudo ser resultado de que estas fueron más abiertas a dar información detallada sobre los sistemas 
de abastecimiento y disposición de agua que se emplean en la vereda Barcelona, en comparación con los hombres (quienes en su mayoría fueron menos expresivos). Las mujeres demostraron percibir con mayor facilidad que los hombres los beneficios que reciben de la naturaleza; además, tal como mencionan Gutiérrez et al. (2013), la mujer (por su rol como administradora del hogar) ha logrado establecer parámetros de calidad de agua, definidos por su experiencia en el uso que le dan al recurso, pues señalan que son las principales usuarias del agua para el uso doméstico, elaboración de alimentos, salud y saneamiento, por lo que perciben mejor los cambios en torno al líquido. A continuación, se muestran las principales fuentes de abastecimiento de agua y la percepción de calidad (Figura 4).

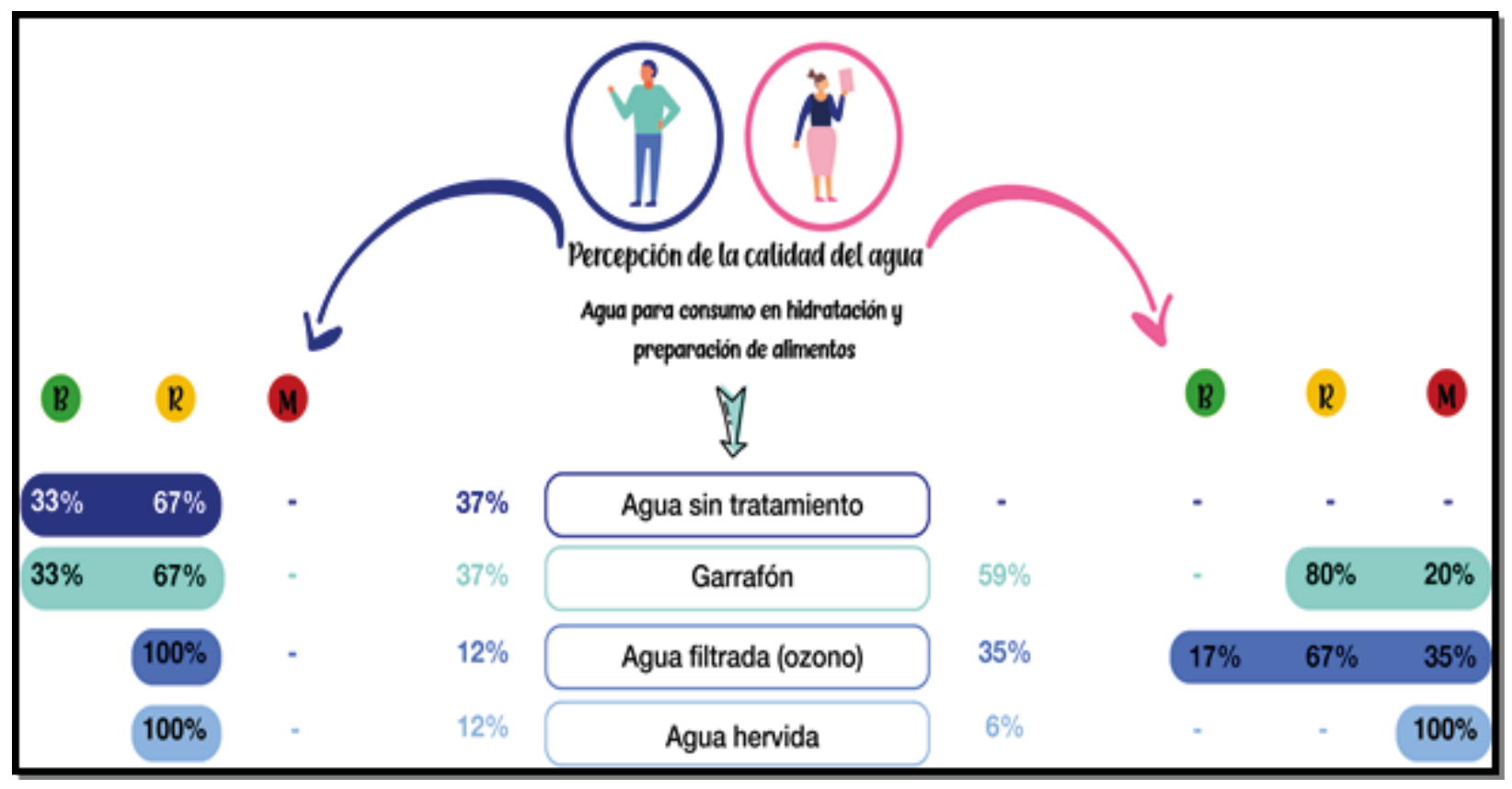

Figura 4. Percepción de la calidad de agua de los hombres y mujeres de la Vereda Barcelona de acuerdo con su tratamiento (para consumo en hidratación y preparación de alimentos). B: Buena, R: Regular, M: Mala.

Fuente: Autores

\subsection{Sistemas de disposición de agua residuales}

Existen diversas tecnologías para tratar las aguas residuales. Estas varían desde las convencionales altamente mecanizadas (que consumen un elevado costo energético), hasta tecnologías ecológicas de bajo costo (Zurita et al., 2011). Actualmente, el porcentaje de aguas residuales municipales (ARM) que se trata en los países en desarrollo apenas ronda el $10 \%$ (Reynolds, 2002); mientras que en América Latina es del $14 \%$, debido principalmente a los altos costos de las plantas de tratamiento (Banco Interamericano de Desarrollo [BID], 2018).

En Colombia el $65 \%$ de los hogares de las zonas urbanas se encuentran conectados a red de saneamiento público, mientras que solo el $27 \%$ de los hogares ubicados en las zonas rurales están conectados (Departamento Nacional de Planeación, 2016). El país se sitúa entre los países latinoamericanos que han extendido su cobertura de tratamiento de aguas residuales domésticas en los últimos años, al lado de Brasil, Chile, Honduras, México, Nicaragua, Perú y Uruguay ( FAO, 2005). En cuanto al alcantarillado, para el año 2016 el departamento del Meta contaba con una cobertura del 58 \% (PNUD, 2020). La comunidad encuestada de la Vereda Barcelona refirió que el $44 \%$ de las viviendas realizan la disposición de aguas residuales en un pozo séptico propio, el $28 \%$ dispone de sus aguas residuales en un pozo séptico y campo abierto, cerca del $20 \%$ realiza la disposición de aguas residuales en la red de 
alcantarillado comunitario y tan solo el $8 \%$ dispone las aguas residuales en la red de alcantarillado comunitario y campo abierto (Figura 5).

En la figura 6, de manera paralela, se muestra una relación entre el nivel de educación de la población encuestada y el distanciamiento entre el pozo séptico y el pozo profundo o aljibe. Se logró identificar que el $50 \%$ de la población que no cuenta con estudios ubica el pozo séptico a una distancia de 1 a 3 metros. La población que cuenta con estudios de básica primaria (14 $\%$ ) ubica el pozo séptico a una distancia de 4 a 6 metros del pozo profundo o "aljibe", el 29 $\%$ a una distancia de 7 a 9 metros y el $57 \%$ a una distancia mayor a 9 metros. La población que cuenta con estudios de bachillerato, técnico y profesional tiene la característica de ubicar el pozo séptico a una distancia mayor de 7 metros del pozo profundo o aljibe, estas personas reconocen la influencia que tiene la distancia entre el pozo séptico y el aljibe para disminuir los riesgos de contaminación y enfermedades causadas por el recurso.

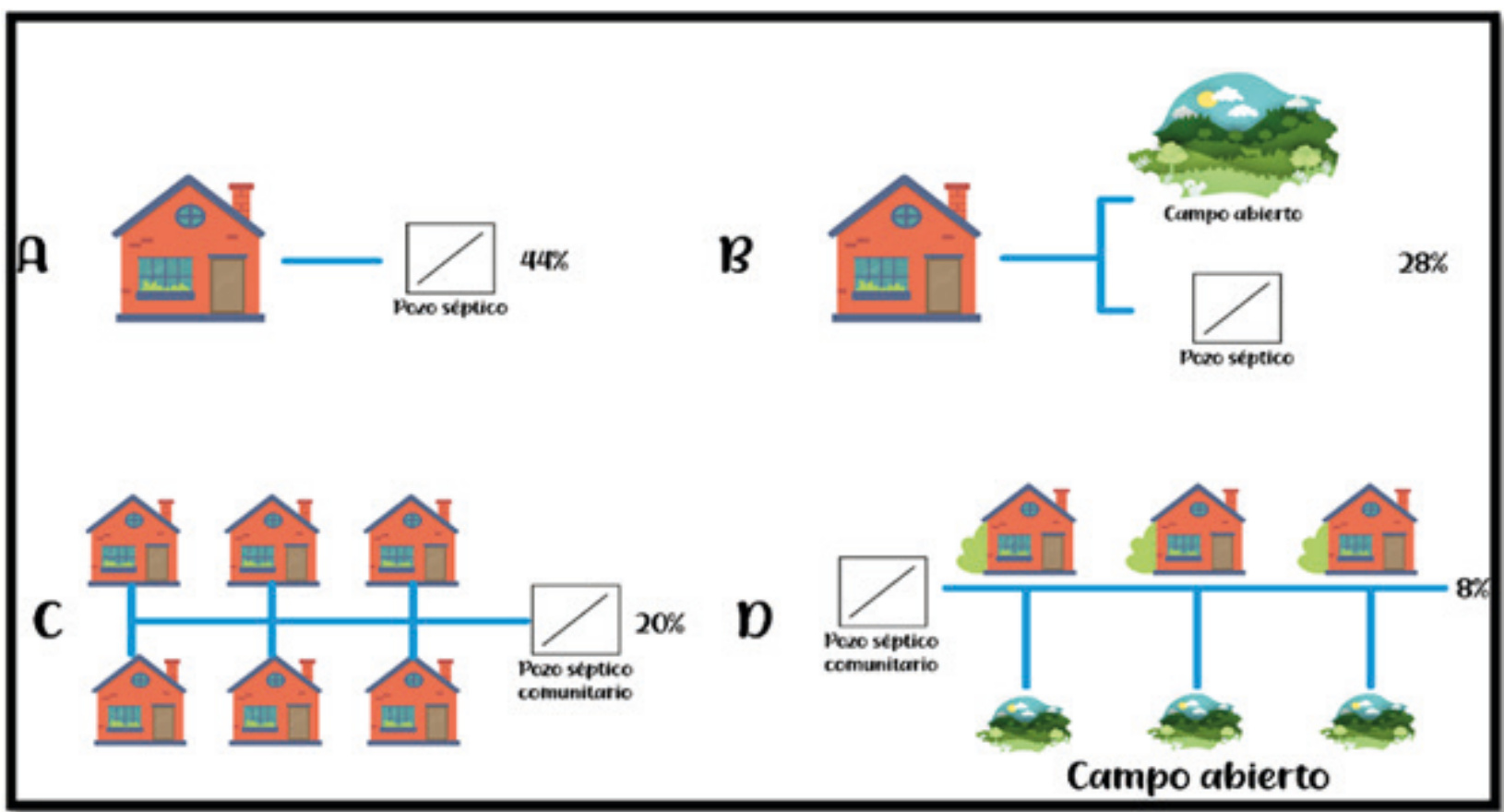

Figura 5. Sistemas de disposición de aguas residuales. A: Disposición de aguas residuales en pozo séptico. B: Disposición de aguas residuales en pozo séptico y campo abierto. C: Disposición de aguas residuales en red de alcantarillado comunitario. D: disposición de aguas residuales en red de alcantarillado comunitario y campo abierto.

Fuente: Autores

Con relación a lo anterior, Rodríguez et al., (2016) reconocen que la solución para disminuir los problemas de salud en comunidades rurales no implica únicamente la ampliación de la cobertura del sistema de acueducto y alcantarillado, requiere mejorar sustancialmente las condiciones higiénicas de las viviendas y garantizar acceso a una adecuada educación académica, ambiental y sanitaria a la población. Adicionalmente, según lo establece el Informe Mundial de las
Naciones Unidas sobre el Desarrollo de los Recursos Hídricos 2021, el acceso seguro al agua y al saneamiento es un derecho legal ( UNESCO, 2021). Por otra parte, Bellido et al. (2010) manifiestan que el nivel de educación tiene una incidencia alta en la prevención de enfermedades relacionadas con el consumo de agua, e identifica que es necesario que la población acceda a educación ambiental y sanitaria para disminuir el riesgo de enfermedades. 


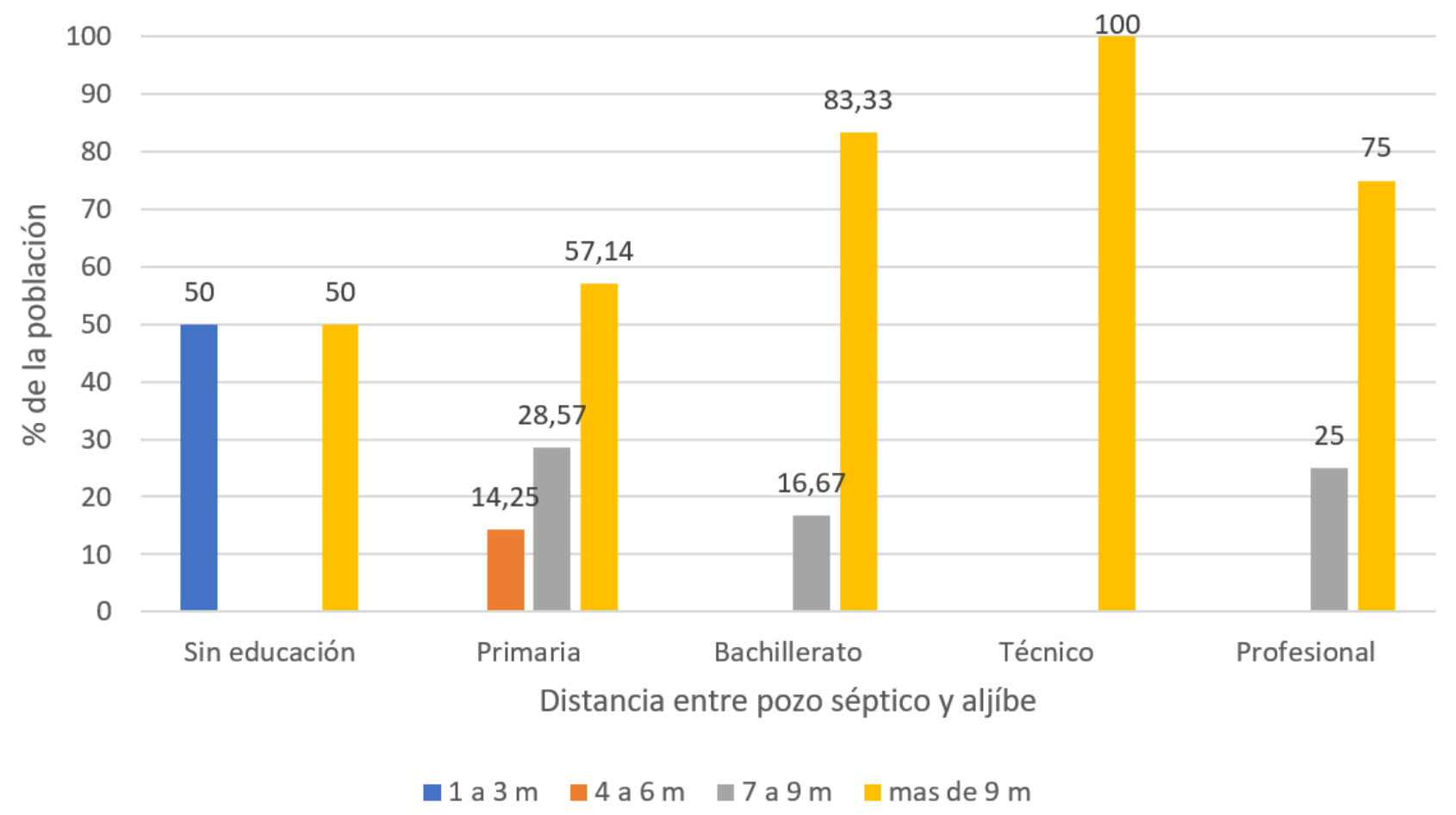

Figura 6. Distancia entre el pozo séptico y el aljibe según el nivel de educación

Fuente: Autores

\section{CONCLUSIONES}

En la vereda Barcelona se encontró que la comunidad se abastece de agua por medio de aljibes y dispone sus aguas residuales mediante cuatro sistemas: pozo séptico; pozo séptico y campo abierto; pozo séptico comunitario; pozo séptico comunitario y campo abierto. A través de la investigación se encontró que la percepción de la calidad del agua varío de acuerdo con género (masculino o femenino), pues en general las mujeres manifestaron tener un mayor conocimiento de los servicios ecosistémicos y del uso del recurso hídrico con respecto a los hombres. De igual manera, se logró identificar que la población que cuenta con estudios de bachillerato, técnico o profesional tienen la característica de ubicar el pozo séptico a una distancia mayor de 7 metros del pozo profundo o aljibe, debido a que estas personas reconocen la influencia que tiene la distancia entre el pozo séptico y el aljibe para disminuir los riesgos de contaminación y enfermedades causadas por el recurso.
Por otro lado, el actual modelo de limpieza de los sistemas de disposición de aguas residuales en la vereda Barcelona es un factor de riesgo, debido a la alta carga de contaminantes aportada por la población del sector. En este escenario, la información derivada de este estudio busca aportar nuevos elementos de análisis que permitan establecer estrategias para la formulación de planes y proyectos encaminados al manejo de los recursos hídricos en esta área rural. Estos deben buscar unificar los criterios para el tratamiento de las aguas obtenidas y la disposición de las aguas residuales, para que estos dejen de ser un factor de riesgo para la salud pública de sus habitantes y el bienestar de los ecosistemas circundantes. Para lograr esto son necesarios estudios de laboratorio que permitan conocer la calidad del agua obtenida, asesorías técnicas a la población por parte de expertos en el tema de gestión del recurso hídrico y programas de educación ambiental. 


\section{CONTRIBUCIÓN DE LA AUTORÍA}

Laura V. Morales-Tejeiro: metodología, investigación, análisisdedatos, conceptualización, escritura, borrador original. Juan M. TrujilloGonzález: investigación, conceptualización, análisis de datos, escritura, revisión y edición. Marco A. Torres-Mora: adquisición de recursos, administrador del proyecto, supervisión, conceptualización, escritura, revisión y edición.

\section{AGRADECIMIENTOS}

Se agradece a los habitantes de la vereda Barcelona por su hospitalidad y disponibilidad para realizar el trabajo de campo, a la Universidad de los Llanos y a su Dirección General de Investigación, a la convocatoria interna Jóvenes Investigadores del 2019, al Semillero y Grupo de Investigación en Gestión Ambiental Sostenible y al Instituto de Ciencias Ambientales de la Orinoquia Colombiana (ICAOC).

\section{LITERATURA CITADA}

Aguilera, A. (2014). Valoración de servicios ecosistémicos de la vegetación urbana en una ciudad desértica. Caso de estudio Ciudad de Antofagasta [Tesis de maestría, Pontificia Universidad Católica de Chile. Chile]. Archivo digital. https://estudiosurbanos. uc.cl/en/exalumnos/valoracion-de-serviciosecosistemicos-de-la-vegetacion-urbanaen-una-ciudad-desertica-caso-de-estudiociudad-de-antofagasta/

Alcaldía de Villavicencio (2015a). Síntesis Diagnostica del Plan de ordenamiento territorial Municipio de Villavicencio. http:// historico.villavicencio.gov.co/Conectividad/ RendiciondeCuentas/Rendici\%C3\%B3n\%20 de $\% 20$ Cuentas $\% 202012-2015 \% 20-\% 20$ Infancia, $\% 20 \mathrm{Adolescencia} \% 20$ y $\% 20$ Juventud.pdf

$\begin{array}{ccc}\text { Alcaldía de Villavicencio } & (2015 b) \text {. } \\ \text { Plan de ordenamiento territorial }\end{array}$ Municipio de Villavicencio. https://www. curaduriasegundavillavicencio.com.co/ normatividad/municipal/POT_2015_ Acuerdo\%20287\%20de\%202015.pdf

Banco Interamericano de Desarrollo. (2018). Proceso regional de las Américas: Foro Mundial del agua 2018. https://www. cepal.org/sites/default/files/news/files/ informe_regional_america_latina_y_caribe. pdf

Barrera, F. D. (2012). La transformación del paisaje rural-urbano y su efecto sobre los servicios ecosistémicos en una microcuenca de Santiago (Chile) [Tesis doctoral, Universidad de Barcelona]. Archivo digital. https://www.researchgate.net/ publication/277720835_La_transformacion_ del_paisaje_rural-urbano_y_su_efecto_ sobre_los_servicios_ecosistemicos_en_una_ microcuenca_de_Santiago_Chile

Bellido, J. G., Barcellos, C., Dos Santos, F. y Bastos, F. (2010). Saneamiento ambiental y mortalidad en niños menores de 5 años por enfermedades de transmisión hídrica en Brasil. Revista Panamericana de Salud Pública, 28, 114-120. https://scielosp.org/ article/rpsp/2010.v28n2/114-120/

Bermúdez, O. B. (2014). La gestión de cuencas hidrográficas en Colombia y su papel en la solución de conflictos por el uso del agua. Caderno Prudentino de Geografía, 1(36), 106-125. https://revista.fct.unesp. br/index.php/cpg/article/view/3175

Brick, T., Gladstone, P., Chandrasekhar, R., Roy, S., Muliyil, J. \& y Kang, G. (2004). Water contamination in urban South India: Household storage practices and their implications for water safety and enteric infections. International Journal of Hygiene and Environmental Health, 207(5), 473-480. https://www.researchgate.net/ publication/8151230_Water_contamination_ in_urban_south_India_Household_storage_ practices_and_their_implications_for_water_ safety_and_enteric_infections

Caro-Caro, C. I., Torres-Mora, M. A. y Barajas-Barbosa, M. P. (2014). Ecosistemas estratégicos y disponibilidad de hábitat de la avifauna del piedemonte llanero (Colombia), como posible peligro aviar. Luna Azul, (39), 25-39. http://www.scielo.org.co/pdf/luaz/ n39/n39a03.pdf

Castro-Garzón, H., Rubio-Cruz, M. A. y Rodríguez-Miranda, J. P. (2013). Análisis y perspectivas de las coberturas de acueducto y alcantarillado en el Departamento del Meta. 
Orinoquía, 18(2), 122-129. http://www. scielo.org.co/pdf/rori/v18n2/v18n2a10.pdf

Chávez, M., Rivera, G. Romero, T. y Vizcarra, I. (2013). El pozo: usos, seguridad y tradición en la subcuenca del río San Javier. Estudios sociales, 21(41), 261-286.

Contraloría General de la Nación (2018). Gestión y Resultados del sector de agua potable y saneamiento básico con énfasis en los recursos del sistema general de participaciones 1994-2017.

https: / / www.contraloria.gov.co/ documents/20181/452124/

Gesti\%C3\%B3n+y+resultados+del+sector+ de+agua+potable+y+saneamiento+ b\%C3\%A1sico+con+\%C3\%A9nfasis+en+ los+recursos+del+sistema+general+de+ participaciones+1994-2017.pdf/572870d8215e-4796-9f03-25509134dddf?version $=1.0$

Delgado-García, S., Trujillo-González, J. y Torres-Mora, M. (2017). Gestión del agua en comunidades rurales; caso de estudio cuenca del río Guayuriba, Meta- Colombia. Luna Azul, (45), 59-70. https://doi.org/10.17151/ luaz.2017.45.5

Departamento Nacional de Planeación (2016). Estudio Sectorial de los servicios públicos domiciliarios de Acueducto $y$ Alcantarillado - 2016. https://www. superservicios.gov.co/sites/default/archivos/ SSPD Publicaciones/Publicaciones/2018/Oct/ estudio_sectorial-compilado-26-12-2017vbibiana.pdf

Faviel, E., Infante, D. y Molina, D. (2018). Percepción y calidad de agua en comunidades rurales del área natural protegida la Encrucijada, Chiapas -México. Revista internacional de contaminación ambiental, 35(2), 317-324. https://doi.org/https://doi. org/10.20937/rica.2019.35.02.05

Fernández, A. (2012). El agua: un recurso esencial. Química Viva, 11(3), 147-170. https://www.redalyc.org/ pdf/863/86325090002.pdf

Gómez, S. y Collazos, J. (2015). Consecuencias del crecimiento demográfico sobre la calidad de la vida en la ciudad de Bogotá, periodo 1985-
2015. [Tesis de pregrado, Universidad de la Salle]. Ciencia Unisalle. https:// ciencia.lasalle.edu.co/cgi/viewcontent. cgi?article $=1288 \&$ context $=$ economia

Gutiérrez, V., Nazar, D., Zapata, E., Contreras, J. y Salvatierra, B. (2013). Mujeres y organización social en la gestión del agua para consumo humano y uso doméstico en Berriozábal, Chiapas. LiminaR 11(2), 100-113. http://www. scielo.org. $\mathrm{mx} / \mathrm{scielo} \cdot \mathrm{php}$ ? script $=\mathrm{sci}$ arttext\&pid=S1665-80272013000200007

Iñiguez , C. D., Bernal, T. y Moreno, J. P. (2015). Correlación entre índices urbanos: La gestión del agua de uso urbano y la marginación urbana. Urbano, 18(32), 50-59. https://www.redalyc.org/ pdf/198/19844017006.pdf

Instituto de Hidrología meteorología y Estudios Ambientales de Colombia (2020). Pronóstico del Tiempo, Villavicencio. http:// www.ideam.gov.co/

Instituto Nacional de Tecnología Agropecuaria (2014). Mantenimiento y tratamiento del agua en aljibes. https://inta. gob.ar/sites/default/files/script-tmp-inta_ mantenimiento_y_tratamiento_del_agua_ en_aljibes.pdf

López, P., y Fachelli, S. (2015). Metodología de la investigación social cuantitativa. Bellaterra (Cerdanyola del Vallès), Universidad Autónoma de Barcelona. http:// infohumanidades.com/sites/default/files/ apuntes/114\%20\%20L\%C3\%B3pez\%20 Rold\%C3\%A1n\%20\%26\%20Fachelli\%20 $\% 20$ An $\%$ C3\%A1lisis $\% 20$ de $\% 20$ tablas $\% 20$ de\%20contingencia.pdf

Martín, L. y Justo, J. (2015). Análisis, prevención y resolución de conflictos por el agua en América Latina y el caribe. https://repositorio.cepal.org/bitstream/ handle/11362/37877/1/S1500220_es.pdf

Mejía, C. (2004). Desafíos para la gestión ambiental frente a la integración económica: El Salvador, Honduras y Nicaragua. http:// www.oas.org/dsd/FIDA/documents/pdf/ estudio_fida.pdf 
Ministerio de Desarrollo Económico (2000). Reglamento técnico del sector de agua potable y saneamiento básico RAS - 2000. https://www.minvivienda.gov.co/ viceministerio-de-agua-y-saneamientobasico/reglamento-tecnico-sector/ reglamento-tecnico-del-sector-de-aguapotable-y-saneamiento-basico-ras

Resolución 2115 de 2007. (2007,4 de julio). Ministerio de la Protección Social. Diario Oficial 46 679. https://www.minambiente.gov.co/ images/GestionIntegraldelRecursoHidrico/ pdf/Legislación_del_agua/Resolución_2115. pdf https://www.alcaldiabogota.gov.co/ sisjur/normas/Norma1.jsp?i=30008

Ochoa, J. M. (2017). Uso y tenencia de la tierra... Factores históricos y planes de ordenamiento territorial- veredas Barcelona, Cocuy y Zuría - Villavicencio Colombia. Revista de Direito Da Cidade, 9(4), 1652-1689. https://doi.org/10.12957/ rdc. 2017.30317

Organización Mundial de la salud (2004). Guías para la calidad de agua potable. https:// www.who.int/water_sanitation_health/dwq/ gdwq3sp.pdf

Organización Mundial de las Naciones Unidas para la alimentación y la agricultura (2005). Objetivos de desarrollo del Milenio. Una mirada desde América Latina $y$ el Caribe. https://www.cepal.org/es/ publicaciones/2797-objetivos-desarrollomilenio-mirada-america-latina-caribe

Organización de las Naciones Unidas para la Alimentación y la agricultura. s.f.(2020). Aquastat. http://www.fao.org/aquastat/es/

Otzen, T. y Manterola, C. (2017). Técnicas de muestreo sobre una población a estudio. International Journal of Morphology, 35(1), 227-231. https://doi.org/http://dx.doi. org/10.4067/S0717-95022017000100037

Padrón, A. C. y Cantú, P. C. (2015). El recurso agua en el entorno de las ciudades sustentables. Cultura Científica y Tecnológica, 6(31). http://erevistas.uacj.mx/ojs/index. php/culcyt/article/view/341/323

Pinilla-Rodríguez, D. E. y TorresSánchez, Y. A. (2019). Gasto público social, el acceso al agua potable y el saneamiento de las poblaciones rurales en América Latina. Revista Latinoamericana de Economía, 50(196), 55-81. https:// doi.org/http://dx.doi.org/10.22201/ iiec.20078951e.2019.196.63499

Programa de Naciones Unidas para el Desarrollo. (2020). META, Retos y Desafíos para el Desarrollo Sostenible.

https://www.co.undp.org/content/colombia/ es/home/library/democratic_governance/ meta--retos-y-desafios-para-el-desarrollosostenible.html

https://www.co.undp.org/content/ dam/colombia/docs/Gobernabilidad/ Publicacionesproyectos/UNDP_Co_GOB_ Publicaciones_FICHA\%20META\% 20 -

$\begin{array}{lllllllllllllll}\% & 2 & 0 & \mathrm{R} & \mathrm{E} & \mathrm{T} & \mathrm{O} & \mathrm{S} & \% & 2 & 0 & \mathrm{Y} & \% & 2 & 0\end{array}$ DESAF\%C3\% $\%$ DOS $\% 20$ PARA \% 20EL \% 20 DESARROLLO.pdf

Programa de Naciones Unidas Para El Desarrollo. (2018). ODS en Colombia: Ios retos para el 2030. https://www.co.undp. org/content/colombia/es/home/library/ods/ ods-en-colombia--los-retos-para-2030.html

Bosch, A. P., Navarrete, F., Vidal, J. L. M., Sánchez, L. M., Martos, F. S., Izquierdo, Á. V., y Martín, W. (1997). La contaminación en los acuíferos del Campo de Dalías y Delta del Andarax (Almería). Recursos naturales y medio ambiente en el sureste peninsular (pp. 363-381). Instituto de Estudios Almerienses. https://dialnet.unirioja.es/descarga/ articulo/2245534.pdf

Quintana, R. (2014). En la gestión colectiva el agua se dona, no se vende. Caso Dosquebradas-Risaralda, Colombia. Realis, 4(1), 186-209. https://periodicos.ufpe.br/ revistas/realis/article/viewFile/8814/8789

Reales, R., De Castro, D. \& Viana, D. (2014). Percepción del agua como Derecho Fundamental: Los efectos producidos por la prestación del servicio de agua potable en los habitantes del municipio de Santa Lucía, Atlántico. Justicia, 26, 69-80. http://www.scielo.org.co/scielo. php ? script $=$ sci_arttext\&pid = S0 124 74412014000200006\&lang $=e s$

Reynolds, K. (2002, octubre). El tratamiento de las aguas residuales en 
Latinoamérica. https://agua.org.mx/wpcontent/uploads/2007/10/Tratamientoaguas-residuales-Latinoamerica.pdf

Rodríguez, J. P, García-Ubaque, C. A. y García-Ubaque, J. C. (2016). Enfermedades transmitidas por el agua y saneamiento básico en Colombia. Revista de salud pública, 18(5), 738-745. http://www. scielo.org.co/scielo.php?pid=S012400642016000500738 \&script $=$ sci_ abstract\&tlng $=e s$

Trujillo-González, J. M., Tovar-Hernández, N., Delgado-García, S., Vargas-Ahumada, D. y Torres-Mora, M. A. (2015). Cuenca alta del río Meta: Una mirada socioambiental a los ríos Guayuriba y Ocoa y al caño QuenaneQuenanito. Universidad de los llanos. https://www.researchgate.net/profile/JuanTrujillo-Gonzalez-2/publication/292980675_ Cuenca_alta_del_rio_Meta_Una_mirada_ socioambiental_a_los_rios_Guayuriba_y_ Ocoa_y_al_Cano_Quenane-Quenanito/ links/56b36ad 08 ae 2 c7d 5 caedb6a/ Cuenca-alta-del-rio-Meta-Una-miradasocioambiental-a-los-rios-Guayuriba-yOcoa-y-al-Cano-Quenane-Quenanito.pdf

Trujillo-González, J. M., MahechaPulido, J. D., Torres-Mora, M. A., Brevik, E. C., Keesstra, S. D., \& Jiménez-Ballesta, R. (2017). Impact of potentially contaminated river water on agricultural irrigated soils in an equatorial climate. Agriculture, 7(7), 52. https://doi.org/https://doi.org/10.3390/ agriculture7070052

Organización de las Naciones Unidas para la Educación, la Ciencia y la Cultura. (2019). Informe Mundial de las Naciones Unidas sobre el Desarrollo de los Recursos Hídricos 2019: No dejar a nadie atrás. In. https://unesdoc. unesco.org/ark:/48223/pf0000367304

Organización de las Naciones Unidas para la Educación, la Ciencia y la Cultura. (2021). Informe Mundial de las Naciones Unidas sobre el Desarrollo de los Recursos Hídricos 2021: El valor del Agua. https://agua.org.mx/wpcontent/uploads/2021/03/375750spa.pdf

Vélez, M. V., Ortiz, C., y Vargas, M. C. (2011). Las Aguas Subterráneas: un enfoque práctico. Ingeominas. https://www2.sgc. gov.co/Publicaciones/Cientificas/NoSeriadas/ Documents/Aguas-subterraneas-enfoquepractico.PDF

Venegas, C., Mercado, M. \& Campos, M. C. (2014). Evaluación de la calidad microbiológica del agua para consumo y del agua residual en una población de Bogotá (Colombia). Biosalud, 13(2), 24-35. http:// www.scielo.org.co/scielo.php?script $=$ sci_ arttext\&pid=S1657-95502014000200003

Villamagua, G. C. (2017). Percepción social de los servicios ecosistémicos en la microcuenca El Padmi, Ecuador. Revista Iberoamericana de Economía Ecológica, 27, 102-114. https://www.raco.cat/index.php/ Revibec/article/view/335096

Zurita, F., Castellanos, O., \& Rodríguez, A. (2011). El tratamiento de las aguas residuales municipales en las comunidades rurales de México. Revista Mexicana de Ciencias Agrícolas, 2(1), 139-150.

h t t p : / / w w w. scielo.org. m x/ s c i e l o.ph p ? p i d = S 2007 09342011000700011\&script=sci_arttext
Conflicto de intereses

Los autores declaran no tener ningún conflicto de intereses.

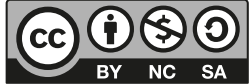

